\title{
Determining the Dust Composition of GRB Host Galaxies
}

\author{
Adria C. Updike*, ${ }^{* a}$ Dieter H. Hartmann ${ }^{b}$ \\ ${ }^{a}$ Roger Williams University \\ Department of Chemistry and Physics \\ One Old Ferry Road \\ Bristol, RI 02809 USA \\ ${ }^{b}$ Clemson University \\ Department of Physics \\ Clemson, SC 29634 USA \\ E-mail: aupdikedrwu.edu, hdieter@clemson.edu
}

\begin{abstract}
Gamma ray burst (GRB) spectral energy distributions (SEDs) are typically fit with a dust extinction template with pre-determined graphite-to-silicate ratios (see Figure 1) to extract the approximate dust composition. These templates are an oversimplification of composition and often rely on non-physical fitting parameters. We instead propose a model that allows for the ratio of graphite to silicate to vary, enabling us to detect smaller amounts of graphite in GRB host galaxies than previously determined with physical model parameters relying on fixed ratios of graphite to silicate column densities. Data from the literature was used to construct GRB SEDs. The optical depths of astronomical silicates and graphite were calculated for a typical size distribution of dust particles and fit to the SEDs using our fitting function. A linear regression produced the best-fit and errors to the dust column densities.
\end{abstract}

Swift: 10 Years of Discovery,

2-5 December 2014

La Sapienza University, Rome, Italy

${ }^{*}$ Speaker. 


\section{Introduction}

Dust extinction produces modifications to the spectral energy distribution (SED) of a gamma ray burst (GRB) along the line of sight through the host galaxy. A GRB SED is a simple combination of power laws caused by synchrotron emission from electrons. The presence of dust in the host galaxy causes wavelength-dependent extinction and can be modeled by a combination of silicate and graphite particles. Current attempts to extract the dust composition of GRB host galaxies have been constrained to particular template models, such as the MW, LMC, and SMC templates with fixed graphite-to-silicate ratios [1], curves with artificial features made to fit observations [2, 3], and curves fit with non-physical parameters $[4,5]$. Our model has several advantages over previous work. First, it does not assume a template; the amount of graphite and silicate are free to vary. Second, it contains no non-physical parameters; each parameter has a physical significance and is the result of observed qualities of the materials. Third, it contains fewer fit parameters than the more complicated of the models mentioned above and can therefore be applied to bursts for which we have fewer data points and can still result in reasonable values for the column densities given a spectral index $\beta$ (taken from Swift XRT data). Fourth, our model has the advantage of not only predicting an extinction value AV but also can determine the column densities of dust along the line of sight as well as a dust-to-gas ratio (given an external measure of the hydrogen column density).

\section{Data Selection}

We began with a sample of 79 GRB SEDs spanning 1997 - 2009. These SEDs were complied from the literature using the methods outlined in [6] and [7] to produce a contemporaneous spectral energy distribution at one day after the trigger including five or more data points (to fit with our four-parameter model) red of the Lyman alpha forest. The methods from [6] and [7] specify that the original data came from the literature; light curves from the literature were fit using a single or broken power-law model (broken in the case of a jet break) to interpolate the magnitude at one day after the burst time in the host galaxy frame, thereby eliminating concerns as to flaring in the data. Our data range from the near-UV to the near-IR and come from a wide variety of instruments. We disregard any data points with a reported error of more than 0.5 magnitudes and with a frequency in the host frame greater than $2.5 \times 10^{15} \mathrm{~Hz}$. Only GRBs for which we have a measured spectroscopic redshift (or excellent photometric redshifts) are included in this sample. To further simplify our model, we then restricted ourselves to bursts for which Swift XRT has a published value for the energy index $(\beta)$ based on the power law electron energy distribution in the $\mathrm{x}$-ray spectrum (average over the data, not at one single time). As we see below, this allowed us to constrict our fit to three free parameters with only the additional choice of whether or not a cooling break in the spectrum (from $\beta_{X}$ to $\beta_{X-0.5}$ ) is justified based on $\chi^{2}$ analysis.

\section{Dust Extinction Model}

The dust compositions considered in this model consist of astronomical silicates (a mix of common silicate oxides) and graphite. The dielectric functions of both materials were taken from laboratory data presented in [8]. Dust particles were assumed to be spherical in shape and range 
between $0.005 \mu \mathrm{m}<a<0.250 \mu \mathrm{m}$ in size with an MRN power law distribution in sizes [9]. The optical depth of each component as a function of frequency was calculated as outlined in [10] for particles ranging from the Rayleigh limit, to Mie scattering, and up to geometric optics (using extinction efficiency coefficient calculations as outlined in [11], [12], [13]). A dust temperature of $20 \mathrm{~K}$ was assumed, but the resulting predictions of the model showed that the temperature dependence of the dust compositions is negligible over the useful range of frequencies (near-UV to near-IR) up to extreme temperatures. The resulting curves for the optical depths of silicate (blue) and graphite (red) assuming a standard column density are given in Figure 1. While the $2175 \AA$ bump is the most prominent feature of the graphite curve, we can also see a distinct curvature of graphite as compared to silicates at smaller wavelengths. This gives us several factors on which the composition of the dust can be determined. Furthermore, within the relevant redshift range of the bursts in our sample, the $2175 \AA$ feature is covered by a standard filter band (see Figure 2).

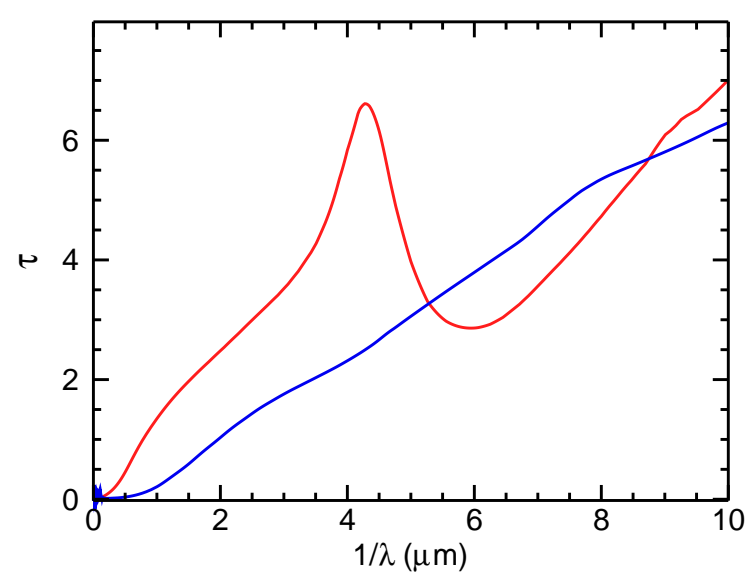

Figure 1: Optical depth divided by column density as a function of wavelength for silicates (blue) and graphite (red) as calculated from our particle distribution.

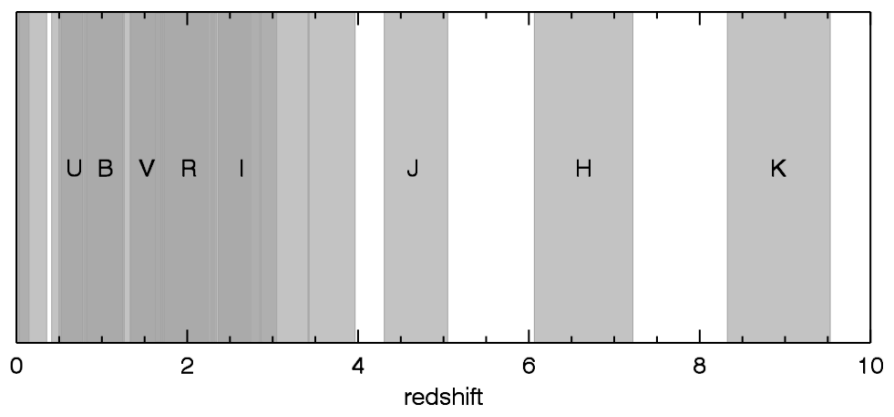

Figure 2: Filter band in which the $2175 \AA$ feature will fall as a function of redshift to the GRB host galaxy.

The fitting function (3.1) to the observed fluxes is adapted from [7] to include the normalizing flux $F_{0}$ and the column densities of graphite and silicate $\left(\Sigma_{S}\right.$ and $\left.\Sigma_{G}\right)$. The optical depths of silicate and graphite $\left(\tau_{S}\right.$ and $\tau_{G}$ ) are calculated as described above, and the value of the energy index $\beta$ is 
determined by the better fit to the data between the choices of $\beta_{X}$ as reported by the Swift XRT team or $\beta_{X-0.5}$ to include a cooling break (the cooling break was preferred in $76 \%$ of cases).

$$
F=F_{0} v^{-\beta} e^{-\left(\Sigma_{S} \tau_{S}+\Sigma_{G} \tau_{G}\right)}
$$

Our data from the SEDs was fit to the above function using a linear regression model resulting in fit parameters (physical parameters of the system) and associated errors.

\section{Initial Results}

Our final sample of GRB hosts consists of 25 SEDs which satisfy the conditions above and produce reasonable fitting results. Unphysical results will be re-analyzed in the future using different dust size distributions to look for the possible "grey" dust claimed to result from supernovae. The results of our fit found that nearly all GRB SEDs had a non-negligible graphite contribution, with $60 \%$ of the bursts fit with a graphite-to-silicate ratio over $10 \%$ (with an average ratio of $16 \%$ ). Compared with similar samples (i.e., the [7] "Golden Sample") we find that GRB SEDs are typically fit with an SMC extinction curve (77\% of sample), consisting of no graphite dust. The LMC extinction model [1] has a graphite-to-silicate ratio of $20 \%$, and the SMC model has a ratio of $0 \%$ (all silicates). With an average ratio of $16 \%$ as found in our sample of 25 bursts, most of the graphite is being missed by using a simple template method.

Sample fits to our SEDs are shown in Figures 3 and 4, representing the highest to some of the lowest graphite-to-silicate ratios. We can see the results of our fit are sensitive to even low amounts of graphite in the host galaxy, allowing for more host galaxy and high redshift graphite detections than found in previous studies.
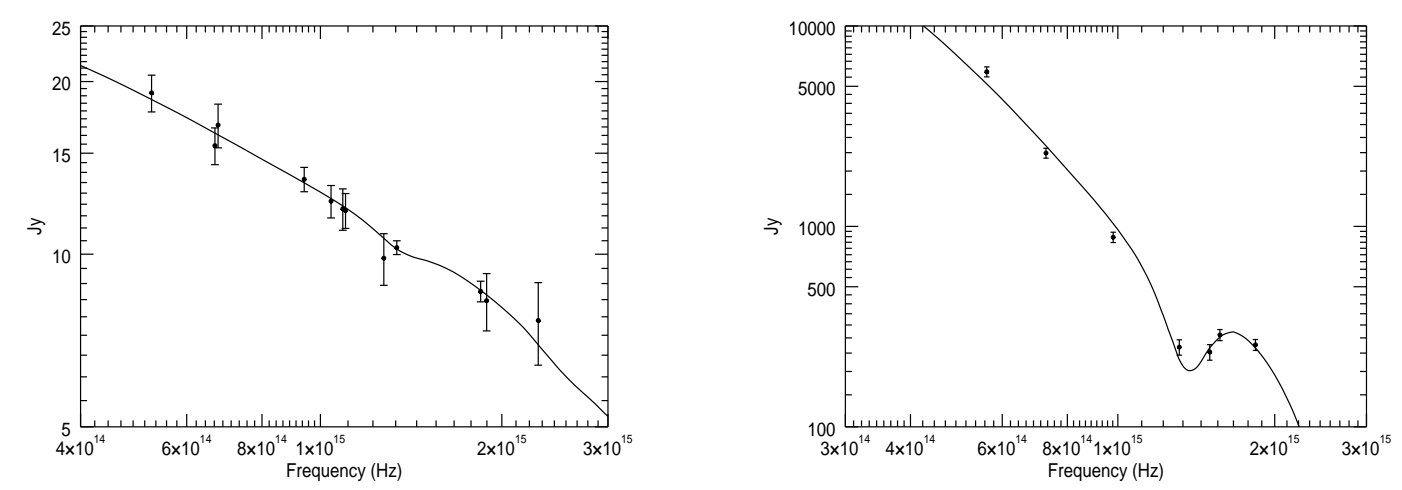

Figure 3: (a) SED of GRB 080514B with a photometric redshift of $z=1.80$ [14] and a graphite-to-silicate ratio of $16.5 \%$, and (b) SED of GRB 080607 with $z=3.036$ and a graphite-to-silicate ratio of 54.2\% (data from [15]). 

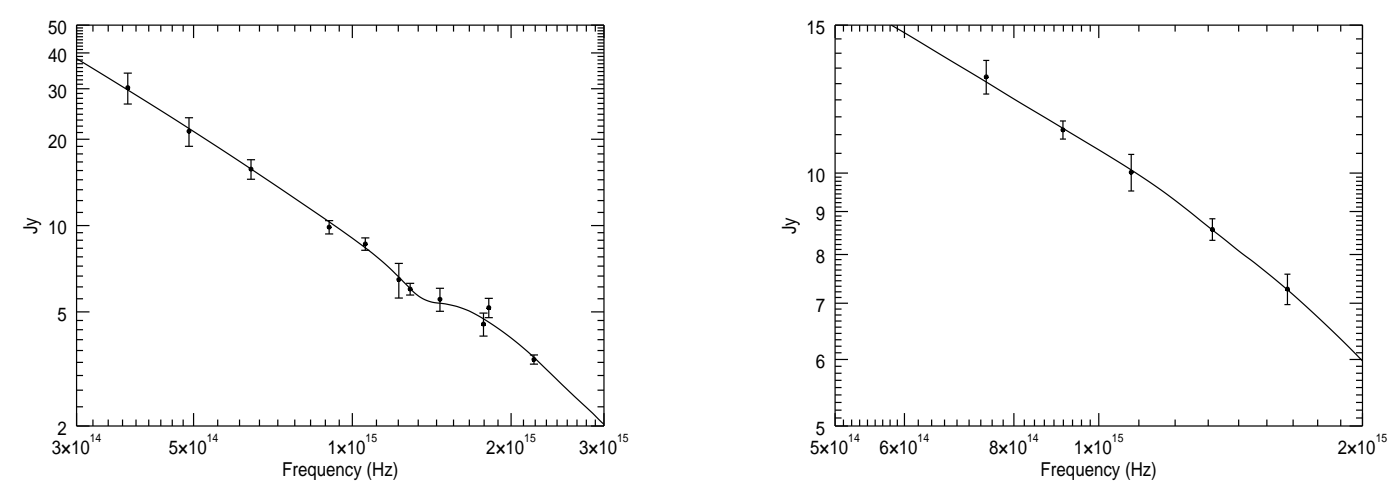

Figure 4: (a) SED of GRB 080928 with $z=1.69$ and a graphite-to-silicate ratio of 4.5\%, and (b) SED of GRB 050802 with $z=1.71$ and a graphite-to-silicate ratio of $3.3 \%$.

\section{Discussion}

The initial results of our study of graphite-to-silicate ratios are shown in the previous section. We find that the host galaxies of GRBs are dominated by silicates, as we would expect from early galaxies dominated by core-collapse supernova (CCSN) dust (i.e., [16]. Graphite (carbon) dust is produced in smaller quantities in most CCSN [17], and is also produced in small quantities by evolving AGB stars [18]. However, no clear evolution in the dust composition is detected in this small sample. We plan to further expand our galaxy evolution models to find out if the overall small graphite-to-silicate ratio can be predicted by a standard Salpeter IMF or if it requires a more top-heavy IMF. Our sample size will also be expanded to include data from 2009 - 2014.

\section{References}

[1] Y. C. Pei, Interstellar dust from the Milky Way to the Magellanic Clouds, ApJ 395 (1992) 130.

[2] E. L. Fitzpatrick \& D. Massa, An analysis on the shapes of ultraviolet extinction curves. I - The 2175 A bump, ApJ 307 (1986) 286F.

[3] J. Cardelli, G. Clayton, \& J. Mathis, The relationship between infrared, optical, and ultraviolet extinction, ApJ 345 (1989) 245C.

[4] D. Calzetti et al., Dust extinction of the stellar continua in starburst galaxies: The ultraviolet and optical extinction law, ApJ 429 (1994) 582C.

[5] Y. Li et al., Determining the Dust Extinction of Gamma-Ray Burst Host Galaxies: A Direct Method Based on Optical and X-Ray Photometry, ApJ 678 (2008) 1136L.

[6] A. Zeh et al., Gamma-Ray Burst Afterglow Light Curves in the Pre-Swift Era: A Statistical Study, ApJ 637 (2006) $889 \mathrm{Z}$. 
[7] D. A. Kann et al., Signatures of Extragalactic Dust in Pre-Swift GRB Afterglows , ApJ 641 (2006) $993 \mathrm{~K}$.

[8] B. T. Draine, Tabulated optical properties of graphite and silicate grains, ApJS 57 (1985) 587D.

[9] J. S. Mathis et al., The size distribution of interstellar grains, ApJ 217 (1977) 425M.

[10] A. G. G. M. Tielens, The Physics and Chemistry of the Interstellar Medium, Cambridge University Press, Cambridge, UK (2005).

[11] C. F. Bohren, Absorption and scattering of light by small particles. Wiley, New York (1983).

[12] A. Laor \& B. T. Draine, Spectroscopic constraints on the properties of dust in active galactic nuclei, ApJ 402 (1993) 441L.

[13] N. C. Wickramasinghe, Light scattering functions for small particles with applications in astronomy, Hilger, London, UK (1973).

[14] A. Rossi et al., A photometric redshift of $z=1.8+0.4-0.3$ for the AGILE GRB 080514B, A\&A 491L (2008) 29R.

[15] D. Perley et al., Monster in the Dark: The Ultraluminous GRB 080607 and Its Dusty Environment, AJ 141 (2011) 36P.

[16] E. Dwek, The Evolution of the Elemental Abundances in the Gas and Dust Phases of the Galaxy, ApJ 501 (1998) 643D.

[17] C. C. Joggerst et al., Mixing in Zero- and Solar-Metallicity Supernovae, ApJ 693 (2009) 1780J.

[18] A. I. Karakas, Updated stellar yields from asymptotic giant branch models, MNRAS 403 (2010) $1413 \mathrm{~K}$. 